\title{
Response of Porcine Articular Cartilage to Irradiation by an Ultrafast, Burst-Mode Laser
}

\author{
Thomas Dzelzainis ${ }^{1}$, Sabrina Hammouti ${ }^{1}$, Melissa Prickaerts ${ }^{2,3}$, Kailas Cassidy ${ }^{2}$, Ömer Ilday ${ }^{4}$, \\ Hamit Kalaycıoğlu ${ }^{4}$, Seydi Yavas ${ }^{5,6}$, Söhret Karamuk ${ }^{6}$, Ahmad Golaraei ${ }^{1,7,8}$, Virginijus Barzda ${ }^{1,8}$, \\ Margarete Akens ${ }^{2,3,9}$, Lothar Lilge ${ }^{3,7}$, and Robin Marjoribanks ${ }^{1}$ \\ ${ }^{1}$ Department of Physics, University of Toronto, ${ }^{2}$ Techna Institute, UHN, ${ }^{3}$ Department of Medical Biophysics, University of Toronto, ${ }^{4}$ Bilkent \\ University, Ankara, Turkey, ${ }^{5}$ Department of Physics, Bogazici University, Istanbul, Turkey, ${ }^{6}$ Lumos Laser, Istanbul, Turkey, ${ }^{7}$ Princess Margaret \\ Cancer Centre, UHN, ${ }^{8}$ Department of Chemical and Physical Sciences, University of Toronto Mississauga, ${ }^{9}$ Department of Surgery, University of \\ Toronto \\ Authore-mail address: tom.dzelzainis@physics.utoronto.ca
}

\begin{abstract}
Plasma-mediated ablation by ultrafast pulses is generally considered to be a material-independent process. We show that, in certain circumstances, this assumption may be invalid. Physical processes involved and the impact on applications are discussed. (C) 2019 The Author(s)
\end{abstract}

OCIS codes: $140.3390,170.1020$

\section{Introduction}

The unique features of the interaction of ultrafast lasers with matter have made them indispensable in the field of materials processing. Unlike long pulse lasers, ultrafast lasers can remove and/or modify material with very little collateral damage or residual heat deposition in surrounding areas. The high intensities reached at focus rapidly ionize and heat a thin layer of material, which lifts off the target surface before significant heat diffuses inward. This makes ultrafast lasers ideal for processing materials in which residual heat can lead to thermal stress and fracture, and biological tissues where tissue denaturation and cell death due to generated heat is a problem with conventional techniques.

There are, however, drawbacks to this approach. High peak intensities needed for initial optical breakdown of dielectric materials result in emission of ionizing radiation and, in certain conditions, production of energetic electrons. Where individual pulse energy is also high, shockwaves may be launched sufficient to rupture tissues or fracture brittle materials. All of which limit the range of applications for which the technique is suitable.

Burst-mode laser processing uses high repetition rate $(>100 \mathrm{MHz})$ pulses delivered in bursts, with a burst containing anywhere from tens to tens-of-thousands of individual femtosecond pulses. The high repetition rate of the pulses within a burst means that the plasma produced by one pulse does not fully recombine between pulses, each pulse is influenced by preceding pulses, and itself influences subsequent pulses. This inter-pulse coupling allows for additional control over the laser-matter interaction not available with single-pulses. For example, some of the undesirable effects stemming from the high intensities needed in single-pulse schemes can be minimized, since only one pulse needs to be intense enough to cause optical breakdown, whilst following pulses can rely on the remaining plasma from the previous pulse to seed the process.

Once an initial plasma is formed, absorption thereafter is assumed to be independent of the material's initial optical properties. The ablation process is therefore considered generic, as all materials are expected to exhibit broadly similar behavior regardless of chemical composition or structure. However, our recent work involving burstmode laser-matter interactions with complex biological materials --which are generally considered as dielectrics when modelling ablation -- has highlighted situations where the assumption of a material independent process seems to be invalid. In this study, we report on the response of porcine articular cartilage to ultrafast burst-mode processing in which we observe behavior indicating departure from the description of the process as generic, plasma-mediated ablation.

\section{Results and Discussion}

Figure 1(a) and (b) show scanning electron microscopy (SEM) images of damage craters formed by single bursts (67 pulses per burst, $200 \mathrm{MHz}$ pulse repetition rate, $300 \mathrm{fs}$ pulse duration, $5 \mu \mathrm{J}$ per pulse), incident on porcine articular cartilage. Figure 1(a) shows a damage crater for a laser incident on superficial-zone cartilage, while figure 1(b) shows the results of a burst incident on transition-zone cartilage, 2-3 $\mathrm{mm}$ below the original surface.

Cartilage exhibits a depth dependent structure reflecting the different functions associated with each zone. The 
superficial zone provides a smooth surface to allow low-friction articulation, and is responsible for most of the tensile properties of cartilage, making it resistant to wear. It is therefore made of tightly spaced collagen fibers lying parallel to the surface. The transition zone is responsible for bearing much of the compressive stress experienced during joint loading; collagen fibers here are less-organized, but generally run oblique to the cartilage surface. Also contained in this extracellular matrix are varying levels of water and other macromolecules.
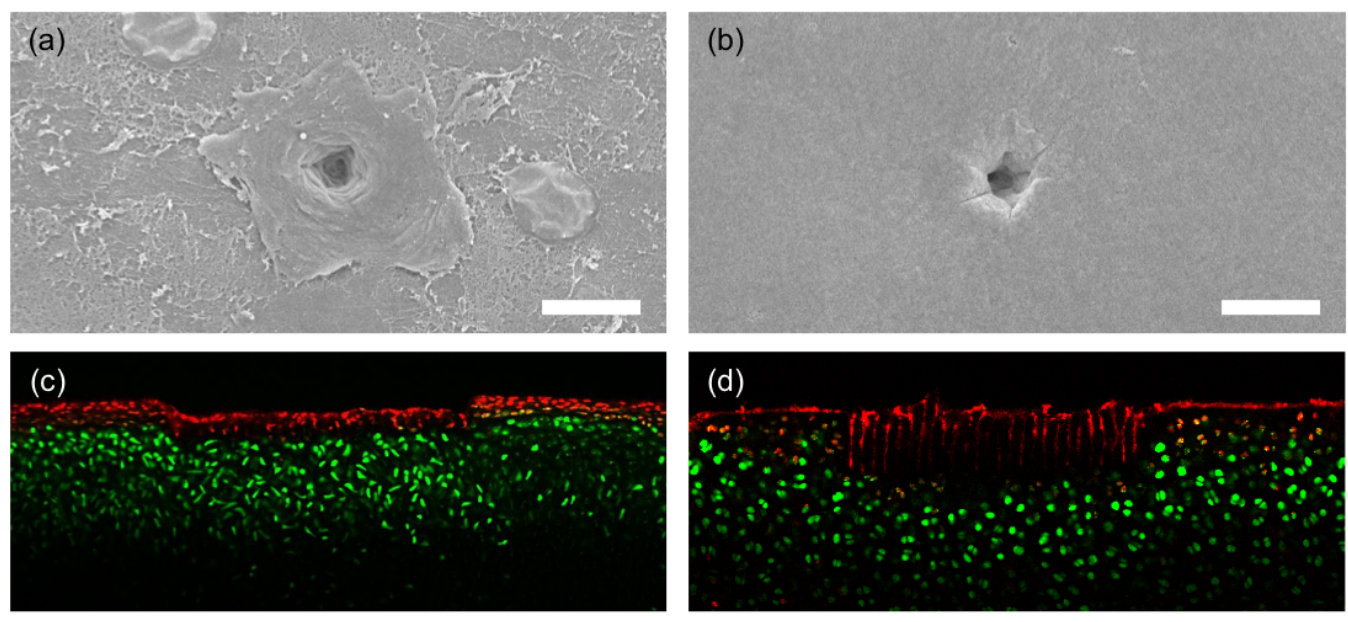

Fig. 1. SEM images of typical damage craters caused by a single burst of pulses incident on (a) superficial-zone cartilage and (b) transition-zone cartilage, scale bars are $10 \mu \mathrm{m}$. (c) Cross-section of an area of transition-zone cartilage processed for material removal by rastering the sample through the laser focus at $2.5 \mathrm{~mm} / \mathrm{s}$. (d) As for (c), but using transition-zone cartilage. In all cases, the laser parameters are the same.

Despite the differing structure and composition of the two interaction sites, the craters show similar diameters of approximately $4 \mu \mathrm{m}$, implying similar breakdown thresholds and similar material-removal rates. Measurements of damage thresholds confirm near identical thresholds for the two cases. This is all as expected from the plasma-mediated description of the process. However, The SEM images show a conspicuous difference in the surrounding tissue damage, with the superficial-zone cartilage showing a large lateral spread of damage, about 20 $\mu \mathrm{m}$ in diameter, while the transition-zone cartilage shows no visible collateral damage. This may be attributable to the differing effect of the small amount of residual heat deposited in the surrounding tissue, although it would require significant differences in the heat transfer properties and/or tissue response to heat in the two zones.

The differences in impact are even more pronounced when a large area of material is processed. Figure 1(c) and (d) show cross-sections of processed areas of (c) superficial-zone cartilage and (d) transition-zone cartilage. In each case, samples were processed by raster scanning over a $2 \mathrm{~mm}$ x $0.5 \mathrm{~mm}$ area with $20 \mu \mathrm{m}$ spacing between consecutive passes. The burst-repetition-rate of the laser is $1 \mathrm{kHz}$, and the sample was moved with a speed of $2.5 \mathrm{~mm} / \mathrm{s}$, meaning bursts were incident on the sample surface at $2.5 \mathrm{um}$ intervals. Samples were then stained with a fluorescent live (green) and dead (red) assay. The processed area was bisected with a scalpel and the cross-section imaged with a scanning confocal fluorescent microscope. The difference between the two cases is immediately clear. In the superficial zone, material is removed to a reasonably uniform depth, with very little cell death below the interaction site. In the transition zone, deep, discrete cuts are made, with damage to twice the depth seen in the superficial zone. In contrast to the superficial-zone case, very little material removal is achieved.

These results show that one of the key features that make ultrafast laser processing so appealing may not always apply in the case of burst-mode lasers. By the use of systematic studies on cartilage, and using comparisons to burst-mode laser ablation of other dielectrics, we investigate the physical processes behind this breakdown, identify potential methods to mitigate or control them, and discuss their impact on the use of ultrafast-burst mode lasers in the processing of biological materials. 